to be reliable and effective in evaluating HPV genotypes in our population. The majority of the HPV genotypes characterised in all genital warts with readouts were either HPV 6 alone or HPV 11 alone or a combination of the two. This supports the use of a HPV vaccine targeting HPV 6 and 11 in the prevention of genital warts in Singapore.

\section{P3.267 WOMEN COLONISED BY LACTOBACILLUS CRISPATUS HAVE A LOWER RISK OF ACQUISITION OF BACTERIAL VAGINOSIS (BV) THAN WOMEN COLONISED BY OTHER LACTOBACILLI}

doi:10.1136/sextrans-2013-051184.0723

'M A Antonio, 'M A Petrina, 'L A Meyn, 1.2S L Hillier. 'Magee-Womens Research Institute, Pittsburgh, PA, United States; 'University of Pittsburgh Department of Obstetrics, Gynecology, and Reproductive Sciences, Pittsburgh, PA, United States

Objective $L$ crispatus, L. jensenii, L. gasseri and L. iners are the predominant lactobacilli in the vaginal flora of reproductive aged women. Colonization of the vagina and rectum by lactobacilli has been associated with decreased risk of BV. We evaluated the speciesspecific role of Lactobacillus on the acquisition of BV.

Methods Two hundred forty four healthy asymptomatic women aged 18-40 were followed at 2 month intervals for up to 18 months. At each visit, vaginal and rectal swabs for culture detection of lactobacilli and a vaginal smear for diagnosis of BV using Nugent criteria were collected. Lactobacilli were identified to the species level using repetitive sequence PCR and/or $16 \mathrm{~S}$ rDNA sequencing. The risk of BV acquisition using Lactobacillus colonisation vaginally and/or rectally as a time-varying covariate was evaluated using Cox proportional hazards models.

Results This analysis included 1481 follow-up visits at which 235 women were colonised by $L$. crispatus, L. jensenii, L. gasseri, or $L$. iners. Of 2734 vaginal and1861 rectal lactobacilli recovered, 1968 were L. crispatus, 1024 L. jensenii, 909 L. gasseri, 410 L. iners, and 284 other species. Eighty nine women acquired BV over 220.4 womanyears (WY) for an incidence of 40 per 100 WY. The rate of BV was lowest among women colonised by $L$. crispatus at the prior visit $(25$ per $100 \mathrm{WY}$, unadjusted hazards ratio $0.31,95 \%$ confidence interval: 0.16-0.62), compared to a rate of 100 per $100 \mathrm{WY}$ among women having only L. iners. Vaginal and/or rectal colonisation by L. jensenii or $L$. gasseri was not associated with lower rates of BV acquisition (60 and 76 per $100 \mathrm{WY}$, respectively $(p>0.05)$ ) than the rate observed among women having only L. iners.

Conclusions Although there is Lactobacillus species diversity in the vaginal microbiome, L. crispatus has the greatest protective benefit against acquisition of BV.

\section{P3.268 A PILOT STUDY OF GENOTYPING EXTRARECTAL LYMPHOGRANULOMA VENEREUM STRAINS}

doi:10.1136/sextrans-2013-051184.0724

'M Vall-Mayans, ${ }^{2} \mathrm{~J}$ Isaksson, 'E Caballero, 'M Barbera, 'M Arando, 'P Armengol, ${ }^{2} \mathrm{~B}$ Herrmann. 'University Hospital Vall d'Hebron, Barcelona, Spain; 'University Hospital, Uppsala, Sweden

Background The first case of LGV in Barcelona was diagnosed in 2005 and since then around 200 cases have been notified up to 2012 All cases have been diagnosed among MSM, $80 \%$ of them coinfected with HIV and $97 \%$ of the cases had proctitis. Since 2008 some cases have appeared with extrarectal manifestations.

Objective To compare the molecular epidemiology profiles of extrarectal LGV cases diagnosed in Barcelona with profiles reported in rectal cases.

Methods A convenient 14 samples from 9 confirmed LGV cases in 2012 with extrarectal involvement were selected for LGV typing. DNA was extracted from samples using a semi automated system and kept al $-80^{\circ} \mathrm{C}$. The strains were further analysed by genotyping using a multilocus sequence typing (MLST) based on 5 highly variable gene regions, in addition the ompA gene was sequenced.

Results DNA quality for MLST was suboptimal in some samples. The genotyping pattern showed one single MLST-5 profile $(27,13$, $17,13,28)$ among all the samples. In ompA there were two variants (22 and 28), in the 2 cases with ompA variant 22 the samples were obtained from inguinal ganglia.

Discussion The MLST-5 profile in LGV cases from Barcelona is the same as the predominating sequence type found in rectal cases. This is in line with the spread of a single clone, without specific tissue tropism. In ompA the 2 cases with variant 22 were identical to ompA in the reference strain $\mathrm{L} 2 / 434 / \mathrm{Bu}$, but differed from the currently predominating variant $\mathrm{L} 2 \mathrm{~b}$ among MSM. Considering the difference in ompA is minor it is more probable that $\mathrm{L} 2 \mathrm{~b}$ is a classical L2 isolate that has been circulating for a long time but showing now a new spectrum of manifestations. Our study does not support any difference in LGV strains obtained from extrarectal sites or from rectum

\section{P3.269 ASSOCIATION OF NEISSERIA GONORRHOEAE NG-MAST STRAIN TYPES AND SPECIFIC MUTATION PATTERN COMBINATIONS IN PENA, MTRR AND PORB}

doi:10.1136/sextrans-2013-051184.0725

'S D Thakur, ${ }^{2 P}$ N Levett, ${ }^{2} \mathrm{G}$ B Horsman, ' $\mathbf{J}$ R Dillon. 'University of Saskatchewan, Saskatoon, SK, Canada; ${ }^{2}$ Saskatchewan Disease Control Laboratory, Regina, SK, Canada

Background Antimicrobial resistance to third generation cephalosporins, penicillin and tetracycline in Neisseria gonorrhoeae isolates can be associated with particular strain types (STs) as well as specific mutation patterns in penA, $m t r R$ or porB. With a view to developing molecular diagnostics for antimicrobial susceptibility, we investigated whether antibiotic resistant and susceptible $N$. gonorrhoeae isolates from Saskatchewan Canada were associated with specific STs and combined mutation patterns in penA, $m t r R$ or por $B$.

Methods DNA sequences of penA, mtrR and porB for $146 \mathrm{~N}$. gonorrhoeae isolates were compared to "wild type" penA (GenBank\#M32091), mtrR (GenBank\#Z25796) and porB (GenBank\#M21289) sequences. Mutation pattern numbers for penA were assigned as described by others. STs were ascertained by NG-MAST. Isolates were selected based on antimicrobial susceptibility phenotypes to 7 antibiotics.

Results Strains were classified into 51 NG-MAST STs; 6 STs (86/146; 59\%) comprised $\geq 5$ isolates, 10 STs included $2-4$ isolates, and 35 STs contained 1 isolate. Isolates with ST 25 (33/36, 92\%) were associated $(\mathrm{P}<0.0001)$ with penA/mtrR/porB pattern I/WT/ WT and with antibiotic susceptibility. ST 3654 was associated $(\mathrm{P}<0.0001)$ with penA/mtrR/porB pattern IX/G45D/G120K,A121D $(\mathrm{n}=13 / 17)$ and CMRNG $(\mathrm{n}=7)$ or CMTR $(\mathrm{n}=6)$ isolates. Isolates with chromosomal resistance to tetracycline were significantly associated $(\mathrm{P}<0.0001)$ with several STs and penA/mtrR/porB patterns including: ST 3655 (XXII/A-,G45D/G120N,A121N - n =8/12), ST 921 (pattern IX/G45D/G120D,A121N - n =6/9), ST 508 (XXII/ G45D/G120D,A121N - n =/6), and ST 3656 (pattern XXII/A-,G45D/ G120D,A121N $-\mathrm{n}=5 / 6$ ). 24 isolates had higher cefixime MICs $(0.03-0.06 \mathrm{mg} / \mathrm{L})$ and included $17 \mathrm{STs}$ with penA pattern IX $(\mathrm{n}=17)$ and $m t r R$ G45D $(\mathrm{n}=16)$ and porB G120K,A121D $(\mathrm{n}=12)$ mutations. Seven of these isolates were associated $(\mathrm{P}<0.0001)$ with ST 3654 (pattern IX/G45D/G120K,A121D).

Conclusions We identified significant associations between particular mutation pattern combinations in pen $A, m t r R$ and por $B$ and specific STs. This indicates that certain combined mutation patterns may be predictive of antimicrobial susceptibility and useful for molecular diagnosis. 